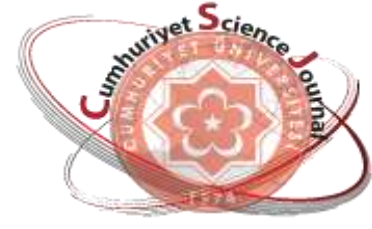

e-ISSN: $2587-246 X$

ISSN: $2587-2680$

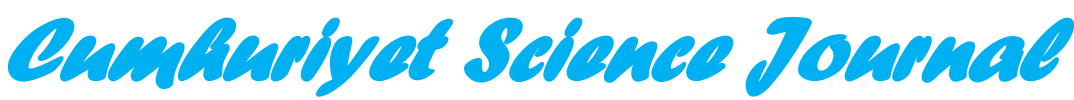

$\operatorname{cs}$

Cumhuriyet Sci. J., Vol.40-1(2019) 173-178

\title{
On the Solution of An Ill-Posed Boundary Value Problem for Second-Order Evolution Equations
}

\author{
İsmet GÖLGELEYEN"(i), Mustafa YILDIZ \\ Zonguldak Bülent Ecevit University, Faculty of Arts and Sciences, Department of Mathematics, Zonguldak,
} TURKEY

Received: 30.10.2018; Accepted: 23.02.2019

http://dx.doi.org/10.17776/csj.476495

\begin{abstract}
In this work, we deal with an ill-posed boundary value problem for multidimensional second-order evolution equations with variable coefficients. By using the given data, we reduce the problem to a functional equation and we obtain a new representation for the solution by means of the Hurwitz formula.

Keywords: Second-order evolution equation, ill-posed problem, functional equation, Hurwitz's formula.

\section{İkinci Mertebeden Evrim Denklemleri için Kötü Konulmuş Bir Sınır Değer Probleminin Çözümü Üzerine}

\footnotetext{
Özet. Bu çalışmada, çok boyutlu değişken katsayılı ikinci mertebeden evrim denklemleri için bir kötü konulmuş sınır değer problemi ele alınmıştır. Veriler kullanılarak problem bir fonksiyonel denkleme indirgenmiş ve Hurwitz formülü yardımıyla bir çözüm elde edilmiştir.

Anahtar Kelimeler: İkinci mertebeden evrim denklemi, kötü konulmuş problem, fonksiyonel denklem, Hurwitz formülü.
}

\section{INTRODUCTION}

In this paper, we present a new representation for the solution of a boundary value problem for multidimensional second-order partial differential equations with variable coefficients. Our method based on the reduction of the problem to a functional equation and use of the Hurwitz formula. We first use our method for an ill-posed problem for the one-dimensional wave equation and then generalize our result for multidimensional second-order differential equations. As an example of recent studies, we refer to [1], where some new representations of the solutions and coefficients of second-order differential equations are given based on the algebraic-analytical identities.

\section{AN ILL-POSED PROBLEM FOR THE ONE-DIMENSIONAL WAVE EQUATION}

In this section, we shall obtain a new representation for the solution of an ill-posed problem for the onedimensional wave equation

$$
\frac{\partial^{2} \omega(x, t)}{\partial t^{2}}=\frac{\partial^{2} \omega(x, t)}{\partial x^{2}},(x, t) \epsilon D \times[0, T],
$$

with the boundary data

$$
\left.\omega(x, t)\right|_{t=0}=s_{0}(x),\left.\omega(x, t)\right|_{t=T}=s_{T}(x), x \in D,
$$


where $D=\left\{x \in \mathbb{R}^{1}:|x|<\tau, 0<\tau\right\}$. It is known that equation (1) has a general solution

$$
\omega(x, t)=f(x+t)+g(x-t)
$$

where $f(x)$ and $g(x)$ are entire functions.

We shall first consider the following boundary value problem:

Problem 1. Find the functions $f(x), g(x), x \in D$ in (3) by data (2).

Theorem 1. Let solution (3) of equation (1) be satisfy boundary data (2). If $s_{0}(x), s_{T}(x), x \in D$ are entire functions, then the formulas

$$
\begin{aligned}
& f(x)=\sum_{k=0}^{\infty} s_{k}(2 T)^{k} \psi_{k}\left(\frac{x}{2 T}\right)+p(x), \\
& g(x)=s_{0}(x)-f(x),
\end{aligned}
$$

are valid, where $s_{k}$ are the coefficients in the power series expansion of $s(x)=s_{T}(x+t)-s_{0}(x)$,

$$
\psi_{k}(x)=\frac{k !}{2 \pi i} \int_{|u|=(2 k+1) \pi} \frac{e^{u x}-1}{e^{u}-1} \frac{d u}{u^{k+1}}, k=0,1,2, \ldots
$$

and $p(x)$ is an arbitrary entire function with period $2 T$.

Proof. By using data (2), (3) we can have following equations for $f(x)$ and $g(x)$

$$
\begin{aligned}
& s_{0}(x)=f(x)+g(x), \\
& s_{T}(x)=f(x+T)+g(x-T),-\infty<x<\infty .
\end{aligned}
$$

In equation (5), we replaced $x$ by $x+T$ to have

$$
s_{T}(x+T)=f(x+2 T)+g(x) .
$$

Then by (4), (6) we obtain the functional equation

$$
f(x+2 T)=f(x)+s(x)
$$

for $f(x)$, where $s(x)=s_{T}(x+T)-s_{0}(x)$.

On the other hand, from Section 8 of Chapter 2 in [2], we know the following result for the functional equations of type (7):

Let the function $f(z)$ be an entire function with $z=x+i y, i^{2}=-1$ such that

$$
f(z+h)=f(z)+a(z), h=h_{1}+i h_{2} \neq 0,
$$

where $a(z)$ is a given entire function. Moreover, we set $a(z)=\sum_{k=0}^{\infty} a_{k} z^{k}$. Then the function

$$
f_{0}(z)=\sum_{k=0}^{\infty} a_{k} h^{k} \psi_{k}\left(\frac{z}{h}\right)
$$

satisfies equation (8), where

$$
\psi_{k}(z)=\frac{k !}{2 \pi i} \int_{|u|=(2 k+1) \pi} \frac{e^{u z}-1}{e^{u}-1} \frac{d u}{u^{k+1}}, k=0,1,2, \ldots .
$$


To obtain the general solution of functional equation (8) in terms of entire functions, it is sufficient to add an arbitrary entire function $p(z)$ with period $h$, that is, $f(z)=f_{0}(z)+p(z)$.

Remark 1. In the special case, if $f(z+1)=f(z)+z^{k}$, then it is easy to find a particular solution of this equation in the form of a polynomial of degree $k+1$. This is the Bernoulli polynomial $B_{k+1}(z)$ which is determined up to a constant term, i.e., $f(z)=B_{k+1}(z)$ and

$$
\frac{e^{u z}-1}{e^{u}-1}=\sum_{k=0}^{\infty} \frac{B_{k+1}(z)}{k !} u^{k},|u|<2 \pi .
$$

Now, let us return to our problem. By Hurwitz's formula (9), the entire particular solution of equation (7) is obtained as follows:

$$
f_{0}(x)=\sum_{k=0}^{\infty} s_{k}(2 T)^{k} \psi_{k}\left(\frac{x}{2 T}\right), T>0,
$$

provided that $s(x)$ in (7) is an entire function, $s(x):=\sum_{k=0}^{\infty} s_{k} x^{k}$, where the functions $\psi_{k}$ are defined in (10). Hence, the general solution of (7) can be written as

$$
f(x)=f_{0}(x)+p(x)
$$

where $p(x)$ is an arbitrary entire function with period $2 T$.

Moreover, the entire function $g(x)$ can be determined from relation (4):

$$
g(x)=s_{0}(x)-f(x)
$$

and so entire solution (3) of equation (1) is obtained.

It is worth noting that we have equations for $f(x), g(x)$ when $x \in D$ only but we can also find $f(z), g(z), z \in \mathbb{C}^{1}$ because $f(z), g(z)$ are entire functions and then $x=\left.z\right|_{y=0}$.

Remark 2. Problem 1 is ill-posed, because

a) The entire data $s_{0}(x), s_{T}(x)$ are determined by only $x \in D$, but in the Hurwitz's formula and formula for the solution $\omega(x, t)$, the continuous extension of these entire data from domain $D$ to the complex space is necessary.

b) The function $p(z)$ is arbitrary periodic and we have nonstability and nonuniqueness.

\section{NEW REPRESENTATION FOR THE SOLUTION OF A GENERAL SECOND-ORDER DIFFERENTIAL EQUATION}

Let $D$ be an open domain in $\mathbb{R}^{n}$, for example $D=\{x:|x|<\tau, 0<\tau\}$. We assume that $a_{i j}(x), a_{i j}=$ $a_{j i}, i, j=1,2, \ldots, n$, are fixed complex valued continuous functions, $v(x) \epsilon C^{2}(D)$ is fixed complex valued function such that

$$
v(x) \neq \text { const, } \sum_{i, j=1}^{n} a_{i j}(x) \frac{\partial v}{\partial x_{i}} \frac{\partial v}{\partial x_{j}} \neq 0, B-C v(x) \neq 0, x \in D,
$$

$B, C$ are constants.

In the future, we shall consider the second-order equation with known complex valued coefficients

$$
\lambda(x) \frac{\partial^{2} \omega}{\partial t^{2}}=\sum_{i, j=1}^{n} a_{i j}(x) \frac{\partial^{2} \omega}{\partial x_{i} \partial x_{j}}+\mu(x) \sum_{i, j=1}^{n} a_{i j}(x) \frac{\partial \omega}{\partial x_{i}} \frac{\partial v}{\partial x_{j}} \equiv L \omega,
$$


where

$$
\lambda(x)=\sum_{i, j=1}^{n} a_{i j}(x) \frac{\partial v}{\partial x_{i}} \frac{\partial v}{\partial x_{j}}, \mu(x)=-\frac{1}{\lambda(x)}\left[\frac{2 C \lambda(x)}{(B-C v(x))}+\sum_{i, j=1}^{n} a_{i j}(x) \frac{\partial^{2} v}{\partial x_{i} \partial x_{j}}\right] .
$$

If the coefficients in (12) are real and $\lambda(x)>0, \sum_{i, j=1}^{n} a_{i j}(x) \xi_{i} \xi_{j} \geq K|\xi|^{2}, K>0$, then (12) is a hyperbolic equation.

In the case of $n=1$ and $a_{11}(x)=a(x)$, equation (12) has a form

$$
a(x)\left(v^{\prime}(x)\right)^{2} \frac{\partial^{2} \omega}{\partial t^{2}}=\frac{\partial^{2} \omega}{\partial x^{2}}-\frac{1}{a(x)\left(\frac{\partial v}{\partial x}\right)^{2}}\left[\frac{2 C a(x)\left(v^{\prime}(x)\right)^{2}}{(B-C v(x))}+a(x) v^{\prime \prime}(x)\right] \frac{\partial \omega}{\partial x} v^{\prime}(x), a(x)\left(v^{\prime}(x)\right)^{2}>0
$$

and if all functions are real, then $\frac{1}{c^{2}(x)}=a(x)\left(v^{\prime}(x)\right)^{2}$ is velocity and $p(x)=$ $-\frac{v^{\prime}(x)}{a(x)\left(\frac{\partial v}{\partial x}\right)^{2}}\left[\frac{2 C a(x)\left(v^{\prime}(x)\right)^{2}}{(B-C v(x))}+a(x) v^{\prime \prime}(x)\right]$ is pressure.

Then we have the following lemma.

Lemma 1. Let $f(z), g(z), z \in \mathbb{C}^{1}$ be arbitrary entire functions. Then the solution $\omega(x, t)$ of (12) has a form

$$
\omega(x, t)=\frac{1}{(B-C v(x))}[f(v(x)+t)+g(v(x)-t)]+\widetilde{\omega}(x, t),
$$

where $\widetilde{\omega}(x, t)$ is a common solution for $n>1$, that is, $\lambda(x) \frac{\partial^{2} \widetilde{\omega}}{\partial t^{2}}=L \widetilde{\omega}$ and $\widetilde{\omega}(x, t)=0$ for $n=1$.

Proof. By relation (13), we have

$$
\begin{aligned}
& \frac{\partial^{2} \omega}{\partial t^{2}}=\frac{1}{(B-C v(x))}\left[f^{\prime \prime}(v(x)+t)+g^{\prime \prime}(v(x)-t)\right]+\frac{\partial^{2} \widetilde{\omega}}{\partial t^{2}} \\
& \frac{\partial \omega}{\partial x_{i}}=\frac{C \frac{\partial v}{\partial x_{i}}[f(v(x)+t)+g(v(x)-t)]}{(B-C v(x))^{2}}+\frac{1}{(B-C v(x))} \frac{\partial v}{\partial x_{i}}\left[f^{\prime}(v(x)+t)+g^{\prime}(v(x)-t)\right]+\frac{\partial \widetilde{\omega}}{\partial x_{i}}
\end{aligned}
$$

and

$$
\begin{aligned}
\frac{\partial^{2} \omega}{\partial x_{i} \partial x_{j}}= & \frac{2 C^{2}(f+g)}{(B-C v(x))^{3}} \frac{\partial v}{\partial x_{i}} \frac{\partial v}{\partial x_{j}}+\frac{C(f+g)}{(B-C v(x))^{2}} \frac{\partial^{2} v}{\partial x_{i} \partial x_{j}}+\frac{2 C\left(f^{\prime}+g^{\prime}\right)}{(B-C v(x))^{2}} \frac{\partial v}{\partial x_{i}} \frac{\partial v}{\partial x_{j}}+\frac{\left(f^{\prime \prime}+g^{\prime \prime}\right)}{(B-C v(x))} \frac{\partial v}{\partial x_{i}} \frac{\partial v}{\partial x_{j}} \\
& +\frac{\left(f^{\prime}+g^{\prime}\right)}{(B-C v(x))} \frac{\partial^{2} v}{\partial x_{i} \partial x_{j}}+\frac{\partial^{2} \widetilde{\omega}}{\partial x_{i} \partial x_{j}} .
\end{aligned}
$$

By (14)-(16), we can see that identity (12) holds.

Now, we shall consider the following boundary value problem.

Problem 2. Find the functions $f(z), g(z), z \in \mathbb{C}^{1}$ in (13) if the data

$$
\left.\omega\right|_{t=0}=\omega_{0}(x),\left.\omega\right|_{t=T}=\omega_{T}(x),\left.\widetilde{\omega}\right|_{t=0}=\widetilde{\omega}_{0}(x),\left.\widetilde{\omega}\right|_{t=T}=\widetilde{\omega}_{T}(x)
$$

are known for $n>1$.

In (17), $\widetilde{\omega}_{0}(x), \widetilde{\omega}_{T}(x), x \in D$, can be regarded as noises. It is clear that $\widetilde{\omega}_{0}(x)=\widetilde{\omega}_{T}(x)=0$ for $n=1$. 
Next, we introduce the functions

$$
S_{0}(x)=\omega_{0}(x)-\widetilde{\omega}_{0}(x), S_{T}(x)=\omega_{T}(x)-\widetilde{\omega}_{T}(x), x \in D,
$$

where

$$
\begin{aligned}
& \left.\widetilde{\omega}\right|_{t=0}=\widetilde{\omega}_{0}(x)=\omega_{0}(x)-\frac{1}{(B-C v(x))}[f(v(x))+g(v(x))] \\
& \left.\widetilde{\omega}\right|_{t=T}=\widetilde{\omega}_{T}(x)=\omega_{T}(x)-\frac{1}{(B-C v(x))}[f(v(x)+T)+g(v(x)-T)] .
\end{aligned}
$$

Moreover, we set

$$
S(v(x))=(B-C(v(x)+T)) S_{T}(v(x)+T)-(B-C v(x)) S_{0}(v(x)):=\left.\sum_{k=0}^{\infty} S_{k} z^{k}\right|_{z=v(x)} .
$$

Hence, we have the following theorem.

Theorem 2. Let solution (13) of equation (12) satisfy data (17). Then the formulas

$$
\begin{aligned}
& f(z)=\sum_{k=0}^{\infty} S_{k}(2 T)^{k} \psi_{k}\left(\frac{z}{2 T}\right)+P(z), \\
& g(z)=(B-C z) S_{0}(z)-f(z),
\end{aligned}
$$

are valid, where $P(z)$ is an arbitrary entire function with period $2 T$.

Proof. By using (13), (17) we can write

$$
\begin{aligned}
& (B-C v(x)) S_{0}(v(x))=f(v(x))+g(v(x)), \\
& (B-C v(x)) S_{T}(v(x))=[f(v(x)+T)+g(v(x)-T)] .
\end{aligned}
$$

In the last equality, we replace $v(x)$ by $v(x)+T$ to have

$$
(B-C(v(x)+T)) S_{T}(v(x)+T)=[f(v(x)+2 T)+g(v(x))] .
$$

Then from (20), (22) we obtain

$$
f(v(x)+2 T)=f(v(x))+S(v(x))
$$

where

$$
S(v(x))=(B-C(v(x)+T)) S_{T}(v(x)+T)-(B-C v(x)) S_{0}(v(x)) .
$$

Finally, taking $v(x)=z$ yields the functional equation

$$
f(z+2 T)=f(z)+S(z)
$$

for $f(z)$ and then by Hurwitz's formula we obtain (18). The entire function $g(z)$ can be calculated by the formula

$$
g(z)=(B-C z) S_{0}(z)-f(z) .
$$

Thus, Theorem 2 is proved. 
Example 1. If $n=1, a_{11}(x)=1, v(x)=x, B=1, C=0, \widetilde{\omega}_{0}(x)=0, \widetilde{\omega}_{T}(x)=0$ in (12), (13), then we have

$$
\frac{\partial^{2} \omega}{\partial t^{2}}=\frac{\partial^{2} \omega}{\partial x^{2}}
$$

and

$$
\omega_{0}(x)=f(x)+g(x), \omega_{T}(x)=f(x+T)+g(x-T), x \in D \subset \mathbb{R}^{1},
$$

for the entire functions $f(z), g(z), z \in \mathbb{C}^{1}$.

Example 2. If $n=3, a_{i j}(x)=\delta_{i j}=\left\{\begin{array}{ll}1, & i=j \\ 0, & i \neq j\end{array}, v(x)=|x|, x \neq 0, B=0, C=-1\right.$, then we have

$$
\frac{\partial^{2} \omega}{\partial t^{2}}=\Delta \omega
$$

and (13) has the following form

$$
\omega(x, t)=\frac{1}{|x|}[f(|x|+t)+g(|x|-t)]+\widetilde{\omega}(x, t),
$$

where $\frac{\partial^{2} \widetilde{\omega}}{\partial t^{2}}=\Delta \widetilde{\omega}$, that is, $\widetilde{\omega}$ is a common solution. Here the boundary value problem is to find the entire functions $f(z), g(z), z \in \mathbb{C}^{1}$ for $S_{0}(x)=\omega_{0}(x)-\widetilde{\omega}_{0}(x)=\frac{1}{|x|}[f(|x|)+g(|x|)], S_{T}(x)=\omega_{T}(x)-$ $\widetilde{\omega}_{T}(x)=\frac{1}{|x|}[f(|x|+T)+g(|x|-T)]$.

Here we note that, in the complex case, all the functions including $v(x)$ are complex and the solution, data and the other functions should be entire. Example 2 is not correct in this case. But in the real case, all the functions are real and in the special hyperbolic case we have different variants. It is clear that $f$ and $g$ must be entire. Example 2 is correct in this case without $x=0$.

\section{Acknowledgments}

We would like to thank Prof. Dr. Yurii E. Anikonov from Sobolev Institute of Mathematics for very fruitful discussions on the problem.

\section{REFERENCES}

[1]. Anikonov, Yu. E., Neshchadim, M. V. Representations for the Solutions and Coefficients of SecondOrder Differential Equations, Journal of Applied and Industrial Mathematics, 7-1 (2013) 15-21.

[2]. Markushevich, A. I. Introduction to the classical theory of Abelian functions, Translations of Mathematical Monographs, 96, American Mathematical Society, Providence, R. I., 1992. 